\title{
Partially-Averaged Navier-Stokes Simulation of the Flow around Simplified Vehicle
}

\author{
S. Krajnović and G. Minelli \\ Department of Applied Mechanics, \\ Chalmers University of Technology, \\ 41296 Gothenburg, Sweden
}

\begin{abstract}
This paper presents Partially-Averaged Navier-Stokes (PANS) simulation of the flow around generic vehicle at different yaw angles. The results of PANS show not only excellent agreement with the experimental data but also large savings in the computer effort compared to LES predictions. Simulations on several computational grids show that PANS adapts to the existing grid making the prediction accurate on affordable computational grids
\end{abstract}

Keywords: Partially-Averaged Navier-Stokes, PANS, bluff body, vehicle

PACS: $47.27 . E-$

\section{INTRODUCTION}

Partially-Averaged Navier-Stokes (PANS) simulations has shown to be an appropriate technique for various bluff body flows of engineering relevance. The applications range from flows around generic bluff bodies such as a finite cylinder [6] to flows around simplified trains [1] and landing gears [2]. One important application of bluff body flow PANS is that of prediction of flow control (passive and active) [3] with the aim of drag reduction in vehicle aerodynamics. The main motivation for our interest in PANS for bluff body aerodynamics is our hope that this technique will be able to overcome the problems with enormous computational effort of large eddy simulation (LES). This paper presents a new application of PANS for a generic vehicle body influenced by crosswind at yaw. The aim of the paper is to explore the capability of PANS to predict this flow. We will rigorously study the prediction of the flow with PANS, compare it with other techniques such as LES, DES and RANS and also explore the capability of PANS to adjust to the existing computational grid.

\section{Partially-Averaged Navier-Stokes}

Partially-Averaged Navier-Stokes (PANS) is a method recently proposed by Girimaji [4] as a bridging technique between RANS and DNS. The switch in PANS is continuous and based on the ratios of unresolved to total kinetic energy and dissipation.

The PANS equations read:

$$
\frac{\partial U_{i}}{\partial t}+U_{j} \frac{\partial U_{i}}{\partial x_{j}}+\frac{\partial \tau\left(V_{i}, V_{j}\right)}{\partial x_{j}}=-\frac{1}{\rho} \frac{\partial p}{\partial x_{i}}+v \frac{\partial^{2} U_{i}}{\partial x_{j} \partial x_{j}}
$$

Here a turbulent velocity $V_{i}$ field is decomposed into two parts by an arbitrary filter as $V_{i}=U_{i}+u_{i} . \tau\left(V_{i}, V_{j}\right)=$ $-2 v_{u} S_{i j}+2 / 3 k_{u} \delta_{i j}$ where $k_{u}$ and $\varepsilon_{u}$ are the unresolved trubulent kinetic energy, and the dissipation and the eddy viscosity of unresolved scales is given as

$$
v_{u}=C_{\mu} \frac{k_{u}^{2}}{\varepsilon_{u}}
$$

in the original PANS $k-\varepsilon$ model by [4]. The resolved stress tensor is given by 


$$
S_{i j}=\frac{1}{2}\left(\frac{\partial U_{i}}{\partial x_{j}}+\frac{\partial U_{j}}{\partial x_{i}}\right)
$$

The model equations for the unresolved kinetic energy $k_{u}$ and the unresolved dissipation $\varepsilon_{u}$ are required to close the system of equation given previously.

The PANS $\zeta-f$ model ([6]) that is used in the present work is given by the following equations:

$$
\begin{gathered}
v_{u}=C_{\mu} \zeta_{u} \frac{k_{u}^{2}}{\varepsilon_{u}} \\
\frac{\partial k_{u}}{\partial t}+U_{j} \frac{\partial k_{u}}{\partial x_{j}}=P_{u}-\varepsilon_{u}+\frac{\partial}{\partial x_{j}}\left[\left(v+\frac{v_{u}}{\sigma_{k u}}\right) \frac{\partial k_{u}}{\partial x_{j}}\right] \\
\frac{\partial \varepsilon_{u}}{\partial t}+U_{j} \frac{\partial \varepsilon_{u}}{\partial x_{j}}=C_{\varepsilon 1} P_{u} \frac{\varepsilon_{u}}{k_{u}}-C_{\varepsilon 2}^{*} \frac{\varepsilon_{u}^{2}}{k_{u}}+\frac{\partial}{\partial x_{j}}\left[\left(v+\frac{v_{u}}{\sigma_{\varepsilon_{u}}}\right) \frac{\partial \varepsilon_{u}}{\partial x_{j}}\right] \\
C_{\varepsilon 2}^{*}=C_{\varepsilon 1}+f_{k}\left(C_{\varepsilon 2}-C_{\varepsilon 1}\right) \\
\frac{\partial \zeta_{u}}{\partial t}+U_{j} \frac{\partial \zeta_{u}}{\partial x_{j}}=f_{u}-\frac{\zeta_{u}}{k_{u}} P_{u}+\frac{\zeta_{u}}{k_{u}} \varepsilon_{u}\left(1-f_{k}\right)+\frac{\partial}{\partial x_{j}}\left[\left(v+\frac{v_{u}}{\sigma_{\zeta u}}\right) \frac{\partial \zeta_{u}}{\partial x_{j}}\right] \\
L_{u}^{2} \nabla^{2} f_{u}-f_{u}=\frac{1}{T_{u}}\left(c_{1}+c_{2} \frac{P_{u}}{\varepsilon_{u}}\right)\left(\zeta_{u}-\frac{2}{3}\right)
\end{gathered}
$$

where constants $C_{\mu}, c_{1}, c_{2}$ and $C_{\varepsilon 2}$ are equal to $0.22,0.4,0.65$ and 1.9 , respectively. $L_{u}$ is the length scale and $T_{u}$ is the time scale defined by using unresolved kinetic energy:

$$
T_{u}=\max \left[\frac{k_{u}}{\varepsilon}, C_{\tau}\left(\frac{v}{\varepsilon}\right)^{1 / 2}\right] ; \quad L_{u}=C_{L} \max \left[\frac{k_{u}^{3 / 2}}{\varepsilon}, C_{\eta}\left(\frac{v^{3}}{\varepsilon}\right)^{1 / 4}\right]
$$

where constants $C_{L}$ and $C_{\tau}$ are equal to 0.36 and 6.0, respectively. We assume that our numerical meshes support the cutoff in the energy containing scales and inertial range and choose $f_{\varepsilon}=1$ (i.e. $\varepsilon_{u}=\varepsilon$ ). $C_{\varepsilon 1}$ is computed as $C_{\varepsilon 1}=1.4\left(1+0.045 / \sqrt{\zeta_{u}}\right)$. This represents the four-equation PANS $\zeta-f$ model with enhanced near-wall attributes.

The unresolved-to-total-kinetic-energy ratio $f_{k}$ in the original PANS model was prescribed as a constant. $f_{k}$ in the PANS $\zeta-f$ model ([6]) to become is a variable parameter that depends upon the grid spacing as follows

$$
f_{k} \geq \frac{1}{\sqrt{c_{\mu}}}\left(\frac{\Delta}{\Lambda}\right)^{2 / 3} ; \Lambda=\frac{k^{3 / 2}}{\varepsilon}
$$

$k$ in Eq. 11 is computed as $k=k_{r}+k_{u}$.

\section{DESCRIPTION OF THE GENERIC CAR AND PREVIOUS INVESTIGATION}

The vehicle studied in the present work was first used in an experimental study of Guilmineau and Chometon [7] who used dynamic differential pressure sensors for steady and unsteady pressure measurements. This flow was later simulated by Guilmineau and Chometon [7] with RANS approach and explicit algebraic stress model (EASM). Guilmineau et al. [8] presented also detached eddy simulation (DES) of this flow. The existence of these RANS and DES simulations together with well documented experimental data makes this flow ideal for evaluation of the capabilities of the present PANS simulations.

The model is shown in Fig.1a. It has a length of $L=675 \mathrm{~mm}$, the width is $W=240 \mathrm{~mm}$, the maximum height is $H=192 \mathrm{~mm}$. All other dimensions of the model are presented in Table. 1 .

The Reynolds number based on the incoming velocity, $U_{\infty}$, and the car length, $L$ used in present paper is $R e=$ $9 \times 10^{5}$. This is the same Reynolds number as in experimental study and RANS simulation of Guilmineau and Chometon [7] and DES of Guilmineau et al. [8]. Three different yaw angles are simulated: $10^{\circ}, 20^{\circ}$ and $30^{\circ}$. The 
a)

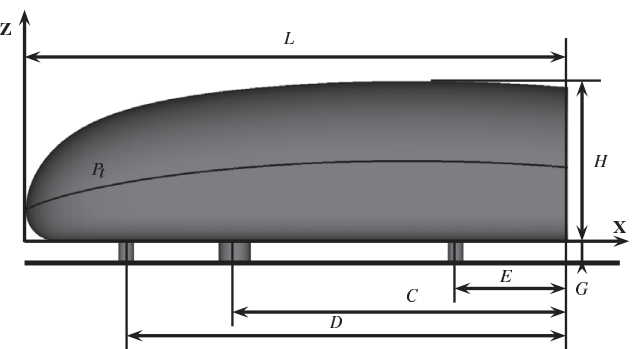

b)

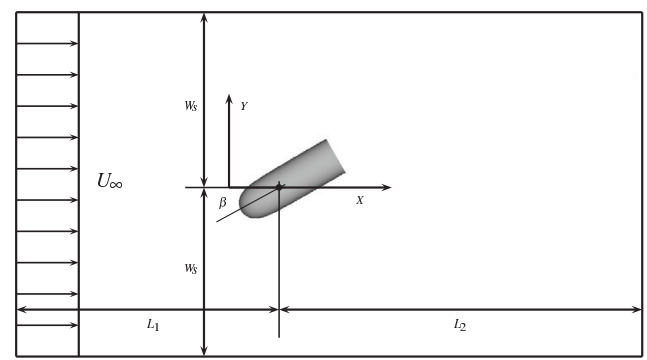

FIGURE 1. a) Geometry of the body. b) Sketch of the models' set-up in the computational domain.

TABLE 1. Dimension of the test model (mm)

\begin{tabular}{ccccccccc}
\hline$C$ & $D$ & $E$ & $F$ & $G$ & $J$ & $K$ & $\phi_{1}$ & $\phi_{2}$ \\
\hline 415 & 550 & 140 & 140 & 29 & 118 & 345 & 20 & 40 \\
\hline
\end{tabular}

model is placed in a domain as shown in Fig. 1b. The average turbulent intensity at the inlet of the wind tunnel used in the experiments of Guilmineau and Chometon [7] was low (2\%). A uniform velocity profile, $U_{\infty}$, constant in time was thus used as the inlet boundary condition in our LES. The homogeneous Neumann boundary condition was used at the downstream boundary. The lateral surfaces and the ceiling were treated as slip surfaces using symmetry conditions for the lateral sides and the ceiling). No-slip boundary conditions were used on the surface of the body and the channel floor.

\section{COMPARISON WITH THE EXPERIMENTAL DATA AND PREVIOUS SIMULATIONS}

Here we only show brief results of the simulations and the future journal paper will include detailed comparison of the results. An example of flow structures resulting from our PANS simulation is shown in Fig. 2. Pressure coefficient

a)

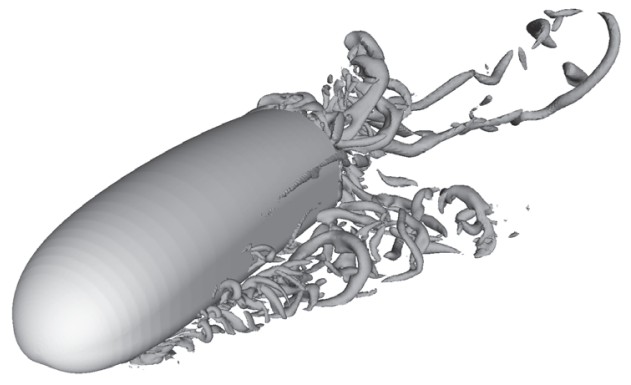

b)

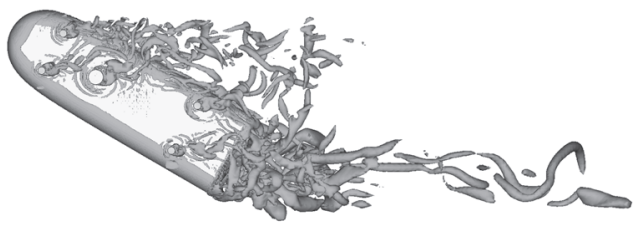

FIGURE 2. An isosurface of the second invariant of the velocity gradient $Q$ for flow at yaw angle $30^{\circ}$. a) Front view. b) View from under the body.

along curve $P_{t}$ for three different yaw angles is compared with the experimental data but also previous LES [9], RANS [7] and DES [8] simulations in Fig. 3. It was found that the $C_{p}$ values of the present PANS agree better with the experimental data than results from previous RANS, DES and LES for all yaw angles. The largest advantage of the PANS over for example LES is for flow on the leeward side of the body and large yaw angles (Fig. 3a). The ability of the PANS to adapt to the available resolution was investigated by running simulation on computation grid containing only half number of the computational nodes compared to the original grid. It was found that the PANS adapts to the grid and increases modelling part accordingly. Fig. 3b shows that the results for the surface pressure remained almost unchanged. This is very interesting finding as the similar exercises with LES would result in drastically change of the results. 

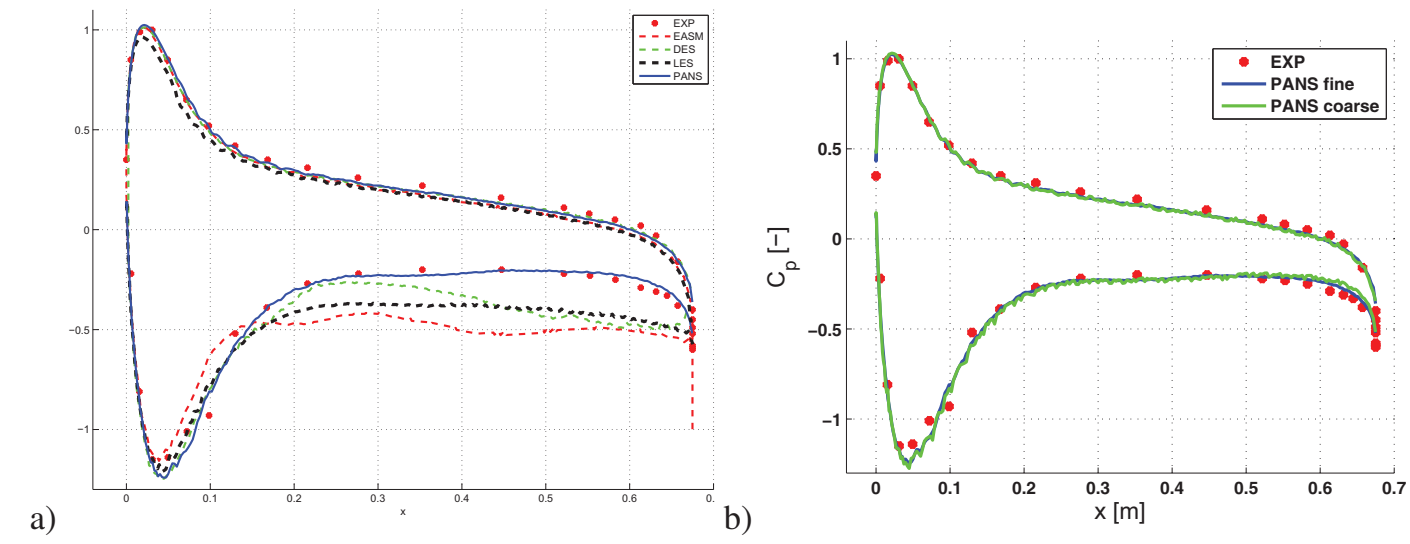

FIGURE 3. a) Pressure coefficient allong curve $\left(P_{t}\right)$ for present LES and PANS and previous RANS [7] and DES [8] for 30 ${ }^{\circ}$ yaw angle. b) Influence of mesh refinement on the PANS results.

\section{CONCLUSIONS}

The PANS model was used successfully to predict the crosswind flow around simplified vehicle. The flow predicted with PANS was found to be in better agreement with the experimental data than predictions of previous LES, DES or RANS simulations. The PANS method was found to adjust to the computational grid by adding or removing turbulence modelling. This increases our hope that PANS can become a true engineering approach for prediction of unsteady flows.

\section{REFERENCES}

1. S. Krajnović and P. Ringqvist and B. Basara, 2012. Comparison of Partially Averaged NavierâĂŞStokes and Large-Eddy Simulations of the Flow Around a Cuboid Influenced by Crosswind, ASME: Journal of Fluids Engineering, Vol. 134.

2. S. Krajnović and R. Lárusson and B. Basara, 2012, Superiority of PANS compared to LES in predicting a rudimentary landing gear flow with affordable meshes, http://dx.doi.org/10.1016/j.ijheatfluidflow.2012.04.013, International Journal of Heat and Fluid Flow.

3. X. Han and S. Krajnović, 2013, Study of active flow control for a simplified vehicle model using the PANS method., International Journal of Heat and Fluid Flow, Vol. 42, pp:139-150.

4. Girimaji, S., Srinivasan, R., Jeong, E., 2003. PANS turbulence models for seamless transition between RANS and LES: Fixed point analyses and preliminary results. In: ASME paper FEDSM2003-4336.

5. Hanjalić, K., Popovac, M., Hadžiabdić, M., 2004. A robust near-wall elliptic-relaxation eddy-viscosity turbulence model for cfd. Int. J. Heat and Fluid Flow 25, 1047-1051.

6. Basara, B., Krajnović, S., Girimaji, S., Pavlović, Z., 2011. Near-Wall Formulation of the Partially Averaged Navier stokes Turbulence Model. AIAA Journal 49 (12), December.

7. E. Guilmineau and F. Chometon, 2009, Effect of side wind on a simplified car model: Experimental and numerical analysis, Journal of Fluids Engineering, Vol. 131.

8. E. Guilmineau and O. Chikhaoui and G. Deng and M. Visonneau, 2009, Effect de vent lateral sur un modele simplifie de voiture par une methods DES, Mecanique \& Industries, Vol. 10, pp: 203-209.

9. S. Krajnović and S. Sarmast, 2010, Numerical investigation of the influence of side winds on a simplified car at various yaw angles,3rd Joint US-European Fluids Engineering Summer Meeting, Symposium on Issues and Perspectives in Ground Vehicle Flows, FEDSM-ICNMM2010-30766, August 1-5, Montreal, Canada. 